TITLE:

\title{
Evaluation of hydrogen bonds of ecdysteroids in the ligand-receptor interactions using a protein modeling system
}

\section{AUTHOR(S):}

Harada, Toshiyuki; Nakagawa, Yoshiaki; Akamatsua, Miki; Miyagawa, Hisashi

\section{CITATION:}

Harada, Toshiyuki ... [et al]. Evaluation of hydrogen bonds of ecdysteroids in the ligandreceptor interactions using a protein modeling system. Bioorganic and Medicinal Chemistry 2009, 17(16): 5868-5873

ISSUE DATE:

2009-08

URL:

http://hdl.handle.net/2433/85254

\section{RIGHT:}

c 2009 Elsevier Ltd. All rights reserved.; This is not the published version. Please cite only the published version.; この論文は出版社版でありませ ん。引用の際には出版社版をご確認ご利用ください。 


\section{Manuscript for Bioorganic Medicinal Chemistry}

Evaluation of hydrogen bonds of ecdysteroids in the ligand-receptor interactions using a protein modeling system

Toshiyuki Harada, Yoshiaki Nakagawa,* Miki Akamatsu, and Hisashi Miyagawa

Division of Applied Life Sciences, Graduate School of Agriculture, Kyoto University Kyoto 606-8502, Japan

* Corresponding author

Yoshiaki Nakagawa, Dr.

Division of Applied Life Sciences

Graduate School of Agriculture

Kyoto University

Kyoto 606-8502

Tel: +81-75-753-6117

Fax: +81-75-753-6123

e-mail: naka@kais.kyoto-u.ac.jp 


\section{Abstract}

The insect molting hormone, 20-hydroxyecdysone (20E) and its analogs (ecdysteroids) specifically bind to the ecdysone receptor. Previously, we synthesized various ecdysteroids containing the side chain moiety of ponasterone A (PonA), and measured the binding activity against Drosophila Kc cells to study the structure-activity relationship. Here we quantitatively analyzed the structure-activity relationship for the ligand binding of ecdysteroids including 20E and PonA. Since the hydrogen bonding (HB) is one of the important physicochemical properties for ligand binding to the ecdysteroid receptor, the number of possible HBs between the ligand molecule and the receptor was manually counted in the modeled ligand-receptor complex for all compounds. The construction of the ligand-receptor model was executed by the full-automatic modeling system (FAMS) in which calculation was done by simulated annealing. The binding potency of 15 ecdysteroids to Kc-cells were linearly correlated $\left(r^{2}=0.63\right)$ with the number of HBs which are observed between ligand and receptor molecule. Contribution of steric and electrostatic effects on the ligand-receptor binding was also examined using a three-dimensional quantitative structure-activity relationship (3-D QSAR), comparative molecular field analysis (CoMFA).

Keywords Ecdysone receptor, Drosophila melanogaster, ponasterone A, hydrogen bond, QSAR, FAMS, CoMFA 


\section{Introduction}

Molting and metamorphosis in arthropods is regulated by a steroidal hormone, usually 20-hydroxyecdysone (20E) ${ }^{1,2}$. The activity of ecdysteroids is mediated by a heterodimer protein complex composed of ecdysone receptor (EcR) and ultraspiracle (USP; a homolog of retinoid X receptor, RXR), which activates the translation of the associated genes after the trigger caused by the binding of the corresponding ligand molecule. Many steroidal as well as non-steroidal compounds have been shown to mimic 20E as a ligand molecule for EcR. In particular, non-steroidal compounds have considerable structural diversity, exemplified by dibenzoylhydrazine (DBH), ${ }^{3,4}$ 3,5-di-t-butyl- 4-hydroxy- $N$-i-butylbenzamide, ${ }^{5} \alpha$-acylaminoketone, ${ }^{6}$ benzoyltetrahydroquinoline, ${ }^{7}$ oxazolines, ${ }^{8}$ and $\gamma$-methylene- $\gamma$-lactams. ${ }^{9} \quad$ Despite such diversity, however, it has been demonstrated that all of the hormonally active compounds, or 'ecdysone agonists' target the same binding site in EcR to mediate their activity, and the structural basis which allows for the activation of a wide range of compounds has attracted attention.

Crystallography-based comparative study of the EcR binding mode between steroidal and non-steroidal ecdysone agonists was first performed by Billas et al. ${ }^{10}$ They demonstrated that the ligand binding domain (LBD) of EcR of the tobacco budworm Heliothis virescens (Lepidoptera; HvEcR) can accommodate both ponasterone A (PonA), one of the most potent steroidal agonists, and a DBH-type non-steroidal agonist, although the corresponding binding pockets were slightly different from each other. Crystal structures of the PonA-bound LBDs have also been solved for EcRs of the sweet potato whitefly Bamisia tabaci (Hemiptera) ${ }^{11}$ and the flour beetle Tribolium castaneum (Coleoptera), ${ }^{12}$ which revealed a substantial similarity in terms of overall 
tertiary structures of LBDs among these three insects. The ligand-receptor interaction was characterized by seven or eight potential hydrogen bonds (HBs) in each of the crystal structures, indicating the importance of the proper spatial arrangement of HB forming groups in an agonist to determine the affinity with the receptor.

Previously, we synthesized a series of ecdysteroid analogues and measured their receptor binding activity in Drosophila Kc cells. ${ }^{13}$ The result showed that the presence of functional groups such as $\mathrm{OH}$ and $\mathrm{C}=\mathrm{O}$ in the ecdysteroids in appropriate positions significantly enhanced the receptor binding. Taking the result of our study into consideration that the presence of multiple $\mathrm{HBs}$ forming groups such as $\mathrm{NO}_{2}$ and $\mathrm{SO}_{2} \mathrm{CH}_{3}$ of DBHs increased the larvicidal activity to Colorado potato beetle Leptinotarsa decemlineata (Coleoptera), ${ }^{14}$ we analyzed the effects of these functional groups in the ecdysteroids on the activity in terms of their HB properties (as HB-acceptors or -donors). However, the total number of HB-forming groups in a molecule was not a sufficient parameter to account for the variation of activity, as exemplified by the difference between ecdysone (E) and PonA. E is about $1000-$ 2400 times less potent than PonA despite having the same number of HB-forming groups present in these two molecules. Therefore, it was thought that whether each of the HB-forming functional groups can effectively form a bond with the acceptor/donor group in the receptor molecule should be taken into consideration to analyze the structure-activity relationship (SAR) in a quantitative manner.

The aim of this study is to examine the effects of HB on the binding of ecdysteroids to receptors. To evaluate the possible number of HBs between ligand molecule and receptor, we constructed a model of the ligand-receptor complex for the LBD of EcR of Drosophila melananogaster (Diptera; DmEcR), which had been 
constructed from the known crystal structure of HvEcR-LBD ${ }^{10}$ by a full-automatic modeling system (FAMS) developed by Ogata and Umeyama. ${ }^{15}$ Then, the binding activity of ecdysteroids to $\mathrm{DmEcR}{ }^{13}$ was quantitatively analyzed using the number of HBs formed in the ligand-receptor complex as a parameter to determine if there was a significant correlation.

\section{Results}

\subsection{Evaluation of hydrogen bonding}

The number of all possible HBs between ligand molecules and the receptor proteins in the virtual ligand-receptor docking models are listed in Table 1. The highest number of HBs (=10) was found in the receptor complex with PonA. Other potent ecdysteroids such as 20E, inokosterone, makisterone and cyasterone formed 7 - 8 HBs with the receptor. Although E carries the same number of functional groups that could be involved in the formation of HBs as PonA, the actual number of HBs found in the E-bound complex was only six. All active compounds had more than four HBs in the complexes, but the numbers of HBs for inactive compounds $8,10,13$ and 15 were all less than three.

The gap between the number of HBs actually found in the complex models and those expected from the number of functional groups of a ligand that can be involved in the formation of HB is typically shown in the constructed models for PonA and 20E (Fig. 1). Although the number of functional groups existing in PonA is six (2,3,14,20,22-OH groups and 6-oxo group), the number of HBs of PonA in the virtual complex is larger than that of $20 \mathrm{E}$, which has seven possible functional groups in the molecule (2,3,14,20,22,25-OH groups and 6-oxo group). A closer examination of each 
model reveals that six amino acid residues (Glu435, Thr467, Arg507, Ala522, Tyr532, Asn628) are commonly involved in the formation of HBs with the ligand molecules, PonA and 20E. Amongst them Tyr532, Thr467, and Ala522 interact with the 20-OH, 14-OH and 6-oxo groups of both steroids, respectively. But the other three interactions are different between 20E and PonA. Glu435 interacts with two OHs at C-2 and C-3, even though the binding modes are different between the two ligand molecules. Arg507 interacts with 2-OH and allows the formation of two HBs for the case of PonA, but only one HB for the case of 20E. Asn628 forms a HB with the 22-OH of PonA and the 25-OH of 20E. In addition to these common amino acid residues, Asn540 and Arg511 form HBs with the 22- and 3-OH groups of PonA, respectively, in a ligand specific manner. Consequently, the number of HBs in the ligand receptor complex is 10 for the case of PonA, and seven for the case of 20E, which does not necessarily reflect the number of functional groups for each ligand.

Thus, the ligand-binding activity of PonA is about 2000-times higher than that of E, though each ecdysteroid has the same number of functional groups. The difference can be accounted for by the actual number of HBs in the virtual model of ligand-receptor complex. The 200-fold enhancement of the activity by the conversion of stereochemistry with respect to A/B ring fusion from trans (16) to cis (17) is inconsistent with a smaller number of $\mathrm{HB}(=5)$ found in the more active compound $\mathbf{1 7}$ compared to that of $16(\mathrm{HB}=6)$. This indicates that other physicochemical properties such as steric and electrostatic effects as well as hydrophobicity are likely to be also important properties for determining the binding activity of a compound, in addition to the HBs. Therefore, the following QSAR analyses were performed to characterize the physicochemical properties that significantly affect the ligand-receptor binding. 


\subsection{QSAR analysis}

The relationship between the binding activity and the sum of HB number was analyzed to derive the statistically significant Eq.1.

$$
\begin{aligned}
& \mathrm{pIC}_{50}= 0.609 \mathrm{HB}+2.583 \\
& \mathrm{n}=15, \quad \mathrm{~s}=0.920, \quad \mathrm{r}^{2}=0.626, \quad \mathrm{~F}_{1,13}=21.753
\end{aligned}
$$

In this and the following equations $\mathrm{n}$ is the number of compounds used to formulate the correlation, $\mathrm{s}$ is the standard deviation, $\mathrm{r}$ is the correlation coefficient, and $\mathrm{F}$ is the F-value of the ratio between regression and residual variances. Although a significant correlation equation was also derived using molecular hydrophobicity log $\mathrm{P}$ as the independent variable instead of $\mathrm{HB}$, it was worse $\left(\mathrm{s}=1.072, \mathrm{r}^{2}=0.491, \mathrm{~F}_{1,13}=12.575\right)$ than Eq. 1 and the coefficient of $\log \mathrm{P}$ was negative $(-0.418)$.

To further examine the effects of steric and other electrostatic factors on the activity, comparative molecular field analysis (CoMFA), ${ }^{16}$ a frequently used three dimensional QSAR technique, was conducted. Equation 2 was formulated using basic CoMFA steric and electrostatic terms as well as the HB term. Even though the basic CoMFA without addition of the HB term yields a significant correlation, the prediction in the cross-validation analysis was worse $\left(\mathrm{q}^{2}=0.381\right.$, Spress $=1.183$, component $\left.=1\right)$.

$$
\begin{aligned}
& \mathrm{pIC}_{50}=2.82+0.549 \mathrm{HB}+[\text { CoMFA steric and electrostatic }] \\
& \mathrm{q}^{2}=0.543 \quad \text { Spress }=1.017, \quad \text { component }=1 \\
& \mathrm{r}^{2}=0.658, \quad \mathrm{~s}=0.880, \quad \mathrm{~F}_{1,13}=24.978
\end{aligned}
$$


(contribution: $\mathrm{HB}=78 \%$, steric= $8 \%$, electrostatic=14\%)

In Eq. $2 \mathrm{q}^{2}$ is the correlation coefficient, and Spress is the standard error for the leave-one-out cross-validation analysis. Increase of components did not improve the correlation in the cross-validation analysis $\left(\mathrm{q}^{2}=0.415\right.$ for component $2, \mathrm{q}^{2}=0.248$ for component 3). Even though the CoMFA hydrogen bonding term is sometimes used instead of $\mathrm{HB},{ }^{17}$ the significant correlation was not formulated $\left(\mathrm{q}^{2}=0.286\right.$, Spress $=1.271$, component=1).

Since the electrostatic features of these compounds are possibly included in HB terms, CoMFA electrostatic term was omitted from basic CoMFA as shown in Eq. 3.

$$
\begin{array}{cl}
\mathrm{pIC}_{50}=2.734+0.585 \mathrm{HB} & +[\text { CoMFA steric }] \\
\mathrm{q}^{2}=0.536 & \text { Spress }=1.025, \quad \text { component }=1 \\
\mathrm{r}^{2}=0.637, & \mathrm{~s}=0.906, \quad \mathrm{~F}_{1,13}=22.835 \\
& \text { (contribution: } \mathrm{HB}=91 \%, \text { steric }=9 \%)
\end{array}
$$

As we expected, the correlation quality did not change significantly by omitting the electrostatic term, and the contribution of the steric effects is similar between Eq. 2 and 3. The CoMFA steric view for Eq. 3 was shown in Fig. 2. Two sterically favorable fields appear near the carbonyl group (C6) and the 2-OH group, and a small sterically favorable field is next to the side chain moiety. An unfavorable field appears above the $\mathrm{B}$ ring along the $\mathrm{C} 7-\mathrm{C} 8$ bond.

Neither correlation of Eq. 2 and 3 are superb, but the variation of activity among the compounds is fairly well accounted for by these equations. The $\mathrm{pC}_{50}$ values 
calculated from Eq. 3 and the deviations between observed and calculated values are listed in Table 1. Even though the activity of three compounds $(\mathbf{1 6}-\mathbf{1 8})$ is not predicted well by Eq. 3, the prediction of other compounds is acceptable. The graphical expression for the relationship between observed and calculated values from Eq. 3 is shown in Fig. 3.

\section{Discussion}

In this QSAR study we estimated the numbers of HBs formed between the ecdysteroids and the molting hormone receptor, using the virtual ligand-receptor complex models constructed in silico. We then tried to relate them to the receptor binding activity. Although we have measured the binding activity of various ecdysteroids using Kc cells derived from Drosophila melanogaster, no 3-D structure of the DmEcR was available, and therefore, we constructed a model from the X-ray crystal structure of HvEcR ${ }^{10}$ using a homology modeling software PDFAMS. ${ }^{15}$ The validity of this methodology has been demonstrated in our previous study, in which the Bombyx mori EcR (BmEcR) was modeled from HvEcR using PDFAMS. ${ }^{18}$ The constructed LBD of the modeled BmEcR was consistent with the structural characteristics deduced from QSAR for the activity of non-steroidal ecdysone agonists such as DBH to receptor. ${ }^{18}$

A Boolean variable designated by integer numbers of 0 and 1 has been widely used as an indicator for the presence of functional groups capable of HB formation in a molecule at specific position in many QSAR studies. In fact, the indicator variable for the presence of HB donating/accepting groups in a molecule was significant in the QSAR for the larvicidal activity of non-steroidal ecdysone agonists against $L$.

decemlineata. ${ }^{14}$ With respect to the receptor binding activity of ecdysteroids, the 
formation of HB was likely to play an important role in complex formation, since the compounds with a relatively small number of HB forming groups had no or very low activity. The use of a widely-used indicator variable appeared to be insufficient to analyze the contribution of HB to the ligand-receptor interactions, since the binding activity of PonA is 2000 times more potent than E, in spite of the presence of the same number of HB-forming functional groups in these two molecules. Therefore, we tried to use the actual number of HB formed in the constructed models of ligand-receptor complexes as a parameter. As exemplified by Fig. 1, some of the HBdonable/acceptable groups of ecdysteroids do not always form HBs, whereas others form multiple HBs per single functional group. As a result, the relatively potent activity of PonA could be explained because it forms the largest number of HBs $(=10)$ with DmEcR. Besides, the numbers of HB predicted in the ligand-receptor complex models for compounds $\mathbf{3}, \mathbf{6}, \mathbf{7}, \mathbf{9}$, and $\mathbf{1 6}$ were larger than those of HB-donable/acceptable functional groups in the respective compounds, whereas the predicted numbers of HB in the complex were smaller than those expected from the numbers of the functional groups, for the cases of $4,8,10,13,15,18$, and 19 . Thus, the number of HB worked fairly well as a parameter in the QSAR analysis for the receptor binding activity of a series of ecdysteroids used in this study. The formulated Eq. 3 indicates that about $64 \%$ of the activity is governed by HB and steric effects, and the activity increases 3.8 times per HB.

Steric effects are probably provided by the distortion of B-ring that was caused by the absence or presence of double bond in the B-ring and the difference of configurations of $\mathrm{A} / \mathrm{B}$ ring fusion. Introduction of the double bond to the B-ring between C7 and C8 makes the ring system planar compared to the saturated B-ring, and 
the direction of $\mathrm{C}=\mathrm{O}$ group slightly changes. The other sterically favorable field near the 3-OH group is dependent on the difference of the A/B ring fusion. The small unfavorable field is probably related to the shift of the C7-C8 bond. Natural ecdysteroids $(\mathbf{1}-\mathbf{6})$ have the double bond between C7 and C8 at B-ring moiety and A/B cis conformation, and these are favorable for activity.

Recently, Browning et al. analyzed the crystal structure of the HvEcR-20E complex. ${ }^{19}$ They found that eight HBs were formed between the ligand and receptor molecules, which was larger by one than the number of HBs in the crystal of complex between HvEcR and PonA (= 7). Thus, they considered that the higher hormonal potency of PonA than 20E can be attributed to the difference in the desolvation energy in the process of the transfer of a molecule from aquatic milieu into the binding pocket in the receptor protein, rather than to the difference in the number of HB between a ligand molecule and the receptor. However, the crystal structure of 20E-HvEcR complex includes a single water molecule that bridges between the ligand and the receptor by HB. Such a water molecule is absent in the crystal of HvEcR-PonA complex, ${ }^{10}$ and therefore these two crystal structures cannot be directly comparable, being inappropriate for the structure-activity study of ecdysteroids.

The hitherto demonstrated structure activity relationships of ecdysteroids are very similar among insect species which is in contrast with SARs of DBHs. ${ }^{20,21}$ Commercial insecticides with non-steroidal structures such as tebufenozide, methoxyfenozide, and chromafenozide are very potent against Lepidoptera, but they are weak or even inactive against other taxonomic insect orders such as Diptera and Coleoptera. Therefore, the discovery of novel structures that mimic the structure of PonA should be fruitful to broaden the spectrum of insecticides. The present QSAR 
study can provide a theoretical basis to design a new ecdysone agonist, which is selectively active against not only Lepidopteran insects, but those of other taxonomic orders.

\section{Conclusion}

Numbers of possible HB between ligand molecules and receptor proteins were evaluated in the ligand-receptor complex models constructed using full-automatic modeling system for each ecdysteroid. The highest number of HBs $(=10)$ is observed for the most potent ecdysteroid, PonA. Compounds having less than three HBs were inactive. The binding of ecdysteroids to the ecdysone receptors of $D$. melanogaster is significantly correlated with the number of HB. Addition of steric effects slightly improved the correlation, even though the contribution of the steric effect was not as large as that of HB according to 3-D QSAR analysis. The HB term evaluated from the ligand-receptor complex is potentially useful for drug design.

\section{Experimental}

\subsection{Compounds and biological activity}

Chemical structures of ecdysteroids and their binding activity are shown in Table 1 . The data for the binding assay with Kc cell extracts were taken from our previous studies ${ }^{4,13 \text {, }}$ 22,23

\subsection{Protein modeling}

EcR-ligand complexes were constructed for all ligand molecules. To construct the LBD of DmEcR, the homology modeling software PDFAMS (Protein Discovery Full 
Automatic Modeling System; In-Silico Sciences, Inc.; Tokyo, Japan) was used. In addition to the basic PDFAMS module, PDFAMS-ligand was used to optimize the structures of ligand-bound receptor proteins.

First, the coordinate of 1R1K, a crystal structure of the domain of the EcR/USP heterodimer of $H$. virescens bound to Pon $\mathrm{A}^{10}$ was downloaded from the PDB web site. Three-dimensional structures of a series of ecdysteroids were constructed by modifying the conformation of PonA bound to the HvEcR LBD and their conformation energy was minimized using PM3 method. Each optimized ecdysteroid was put in the PonA bound cavity to minimize the root mean square (RMS) deviation for the superposition between PonA and each ecdysteroid as described in Section 5.3. The primary sequence of the LBD of DmEcR ${ }^{24}$ was aligned with that of HvEcR using RPS-BLAST as shown in Fig. 4 in order to replace the HvEcR-LBD with the DmEcR-LBD of the constructed model while accommodating each minimized steroidal compound. The sequence identity between DmEcR and HvEcR was calculated to be 67.4\%. The structure of each ligand-bound DmEcR-LBD was optimized by simulated annealing method of PDFAMS-ligand. ${ }^{15}$ The energy of the ligand-DmEcR complex was then minimized using MMFF94 of SYBYL under the limited conditions in which the main chain of protein is fixed. Finally, whole DmEcR-LBD proteins containing hydrogen atoms were subjected to the MMFF94 calculation in order to obtain the most stable conformation of the complex. The numbers of HBs between the ligand molecule and the receptor were manually counted for each ligand-receptor complex. In this counting, HB is determined based on the hydrogen-acceptor distance and the donor-hydrogen-acceptor angle $\left(0.80-2.80 \AA,-120^{\circ}\right.$ to $\left.+120^{\circ}\right)$. 


\subsection{QSAR analysis}

Classical QSAR analysis was performed by QREG2.05, ${ }^{25}$ and 3-D QSAR analysis was done by CoMFA, submodule in SYBYL (6.91). All compounds were superimposed based on a minimal RMS deviation fit to 13 carbon atoms (C8, C9, C11-C17, C18, C20-C22) of PonA by constructing C/D fused ring and side chain moieties as shown in Fig. 5

\section{Acknowledgments}

Our sincere thank to Dr. Toshio Tsuchiya of SGI Japan for the technical support for the running of PDFAMS. We also thank Dr. Vincent Henrich for reviewing the manuscript. The study was supported, in part, by the 21st century COE program for Innovative Food and Environmental Studies Pioneered by Entomomimetic Sciences, from the Ministry of Education, Culture, Sports, Science and Technology of Japan. Toshiyuki Harada is a recipient of a Research Fellowship of the Japan Society for the Promotion of Science for Young Scientists. 


\section{References}

1. Dinan, L. Phytochemistry 2001, 57, 325.

2. $\quad$ Gilbert, L. I.; Warren, J. T. Vitam Horm 2005, 73, 31.

3. $\quad$ Dhadialla, T. S.; Carlson, G. R.; Le, D. P. Annu. Rev. Entomol. 1998, 43, 545.

4. $\quad$ Nakagawa, Y. Vitam Horm 2005, 73, 131.

5. Mikitani, K. Biochem. Biophys. Res. Commun. 1996, 227, 427.

6. Tice, C. M.; Hormann, R. E.; Thompson, C. S.; Friz, J. L.; Cavanaugh, C. K.; Saggers, J. A. Bioorg. Med. Chem. Lett. 2003, 13, 1883.

7. Smith, H. C.; Cavanaugh, C. K.; Friz, J. L.; Thompson, C. S.; Saggers, J. A.; Michelotti, E. L.; Garcia, J.; Tice, C. M. Bioorg Med Chem Lett 2003, 13, 1943.

8. Hormann, R. E.; Chortyk, O.; Le, D. P. Canada Patent, CA $2516270 A 12005$.

9. Liepa, A. J.; Johnson, W. M.; Turner, K. A., PCT Int. Appl. WO 2008 70,891 (Cl.A01N43/34) 2008.

10. Billas, I. M. L.; Iwema, T.; Garnier, J. M.; Mitschler, A.; Rochel, N.; Moras, D. Nature 2003, 426, 91.

11. Carmichael, J. A.; Lawrence, M. C.; Graham, L. D.; Pilling, P. A.; Epa, V. C.; Noyce, L.; Lovrecz, G.; Winkler, D. A.; Pawlak-Skrzecz, A.; Eaton, R. E.; Hannan, G. N.; Hill, R. J. J. Biol. Chem. 2005, 280, 22258.

12. Iwema, T.; Billas, I. M.; Beck, Y.; Bonneton, F.; Nierengarten, H.; Chaumot, A.; Richards, G.; Laudet, V.; Moras, D. Embo J 2007, 26, 3770.

13. Arai, H.; Watanabe, B.; Nakagawa, Y.; Miyagawa, H. Steroids 2008, 73, 1452.

14. Nakagawa, Y.; Smagghe, G.; Kugimiya, S.; Hattori, K.; Ueno, T.; Tirry, L.; Fujita, T. Pestic. Sci. 1999, 55, 909.

15. Ogata, K.; Umeyama, H. J Mol Graph Model 2000, 18, 258.

16. Cramer III, R. D.; Patterson, D. E.; Bunce, J. D. J. Am. Chem. Soc. 1988, 110, 5959.

17. Hormann, R. E.; Smagge, G.; Nakagawa, Y. QCR 2008.

18. Wheelock, C. E.; Nakagawa, Y.; Harada, T.; Oikawa, N.; Akamatsu, M.; Smagghe, G.; Stefanou, D.; Iatrou, K.; Swevers, L. Bioorg Med Chem 2006, 14, 1143.

19. Browning, C.; Martin, E.; Loch, C.; Wurtz, J. M.; Moras, D.; Stote, R. H.; Dejaegere, A. P.; Billas, I. M. J Biol Chem 2007, 282, 32924.

20. Minakuchi, C.; Ogura, T.; Miyagawa, H.; Nakagawa, Y. J. Pest. Sci. 2007, 32.

21. Nakagawa, Y.; Smagghe, G.; Hormann, R. In Ecdysones; Structures and Functions; Smagghe, G., Ed.; Springer, 2009, 475.

22. Nakagawa, Y.; Minakuchi, C.; Takahashi, K.; Ueno, T. Insect Biochem. Mol. Biol. $2002,32,175$. 
23. Watanabe, B.; Nakagawa, Y.; Ogura, T.; Miyagawa, H. Steroids 2004, 483.

24. Koelle, M. R.; Talbot, W. S.; Segraves, W. A.; Bender, M. T.; Cherbas, P.; Hogness, D.

S. Cell 1991, 67, 59 .

25. Asao, M.; Shimizu, R.; Nakao, K.; Fujita, T. JCPE Registered Program No. 115, Japan Chemistry Program Exchange, "Society for Computer Chemistry, Japan. 
Table 1. Ligand binding activity in Kc cells and the number of hydrogen-bonds formed between the compound and the modeled DmEcR-LBD

\begin{tabular}{|c|c|c|c|c|c|}
\hline \multirow[b]{2}{*}{ No. } & \multirow[b]{2}{*}{ Compound } & \multicolumn{3}{|c|}{$\mathrm{pIC}_{50}(\mathrm{M})$} & \multirow[b]{2}{*}{$\mathrm{HB}$} \\
\hline & & Obsd & Calcd $^{\mathrm{a}}$ & $\Delta^{\mathrm{b}}$ & \\
\hline $1^{c}$ & & $5.59^{\mathrm{d}}$ & 6.27 & -0.68 & 6 \\
\hline $2^{e}$ & & $7.34^{\mathrm{d}}$ & 6.87 & 0.47 & 7 \\
\hline $3^{f}$ & & $8.89^{d}$ & 8.66 & 0.23 & 10 \\
\hline $4^{\mathrm{f}}$ & & $7.21^{\mathrm{d}}$ & 6.94 & 0.27 & 7 \\
\hline $5^{h}$ & & $6.95^{\mathrm{d}}$ & 6.88 & 0.07 & 7 \\
\hline $6^{i}$ & & $7.04^{\mathrm{d}}$ & 7.47 & -0.43 & 8 \\
\hline 7 & & $4.38^{j}$ & 4.98 & -0.61 & 4 \\
\hline
\end{tabular}


8

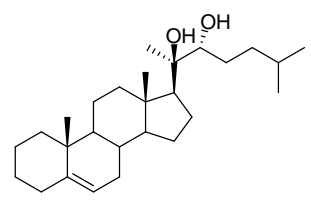

$<3.61^{\mathrm{j}}$

10

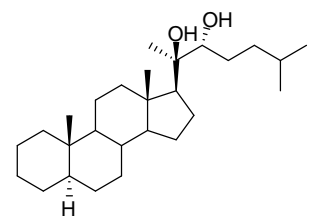

11

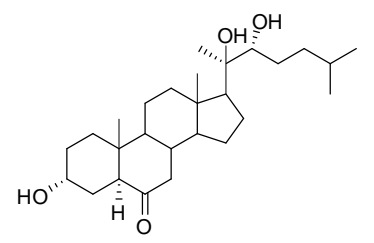

12

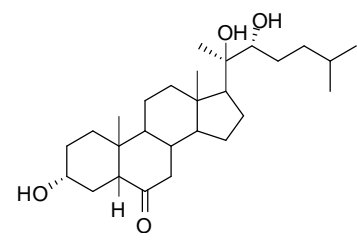

13

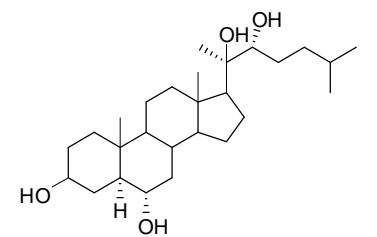

14

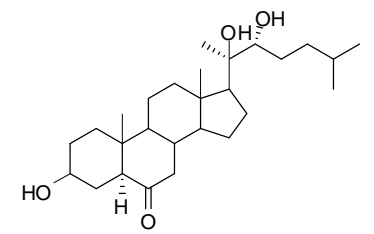

15<smiles>CC(C)CCC(O)C1(C)CCC2C3CCC4CC(O)CCC4(CC3)CCC21</smiles>

$4.38^{\mathrm{j}}$

$<3.61^{\mathrm{j}}$

3.35

1

$\begin{array}{lll}3.35 & - & 1\end{array}$

$6.10^{\mathrm{j}}$

5.03

1.07

4

$4.05^{\mathrm{j}}$

5.05

$-1.00$

4

$<3.61^{\mathrm{j}}$

4.41

3

$5.02^{\mathrm{j}}$

4.98

0.04

4

$<3.61^{\mathrm{j}}$

4.52

3 
16<smiles>CC(C)CCC(O)C(C)(O)C1CCC2C3CC(=O)[C@@H]4CC(O)C(O)CC4(C)C3CCC21C</smiles>

17

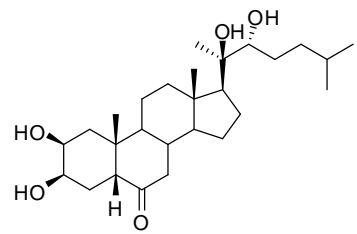

18<smiles>CC(C)CCCC(O)(O)[C@H]1CCC2C3CC(=O)[C@H]4C[C@@H](O)[C@@H](O)CC4(C)C3CCC21C</smiles>

19 $4.84^{\mathrm{j}}$

$7.23^{\mathrm{j}}$

5.64

1.59

5

$6.49^{\mathrm{k}}$

5.02

1.47

4

$4.41^{\mathrm{k}}$

5.01

$-0.60$

4

a) Calculated by Eq. 3. b) Differences between observed and calculated values. c) Ecdysone. d) Ref. [22]. e) 20-Hydroxyecdysone. f) Ponasterone A. g) Cyasterone. h) Makisterone A. i) Inokosterone. j) Ref. [13]. k) Ref. [23]. 


\section{Figure Legends}

Fig. 1. Hydrogen bonds observed between the modeled DmEcR and ligands; (A) Ponasterone A, (B) 20-Hydroxyecdysone

Fig. 2. Stereoview of the CoMFA steric field with PonA for Eq. 3. The contours are shown to surround regions where a higher steric bulk increase (green) or decrease (yellow) the binding.

Fig. 3. Graphical presentation of observed $\mathrm{pIC}_{50}$ values versus $\mathrm{pIC}_{50}$ values calculated by Eq. 3.

Fig 4. Alignment of primary sequences of ligand binding domains of HvEcR and DmEcR by RPS-BLAST. Identical amino acid residues are marked with yellow color.

Fig. 5. Superposition for all 19 compounds. 


\section{Fig. 1}

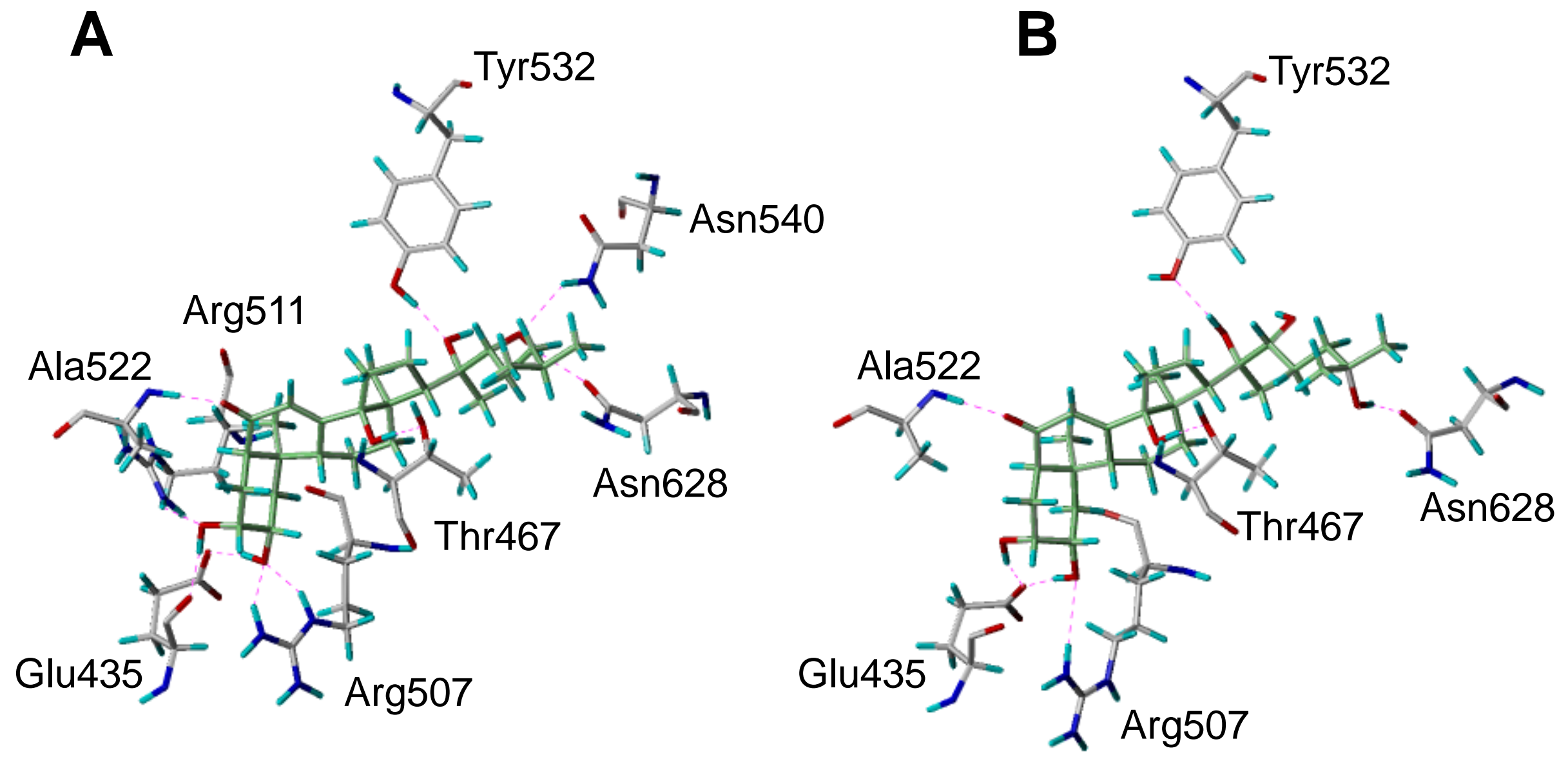




\section{Fig. 2}
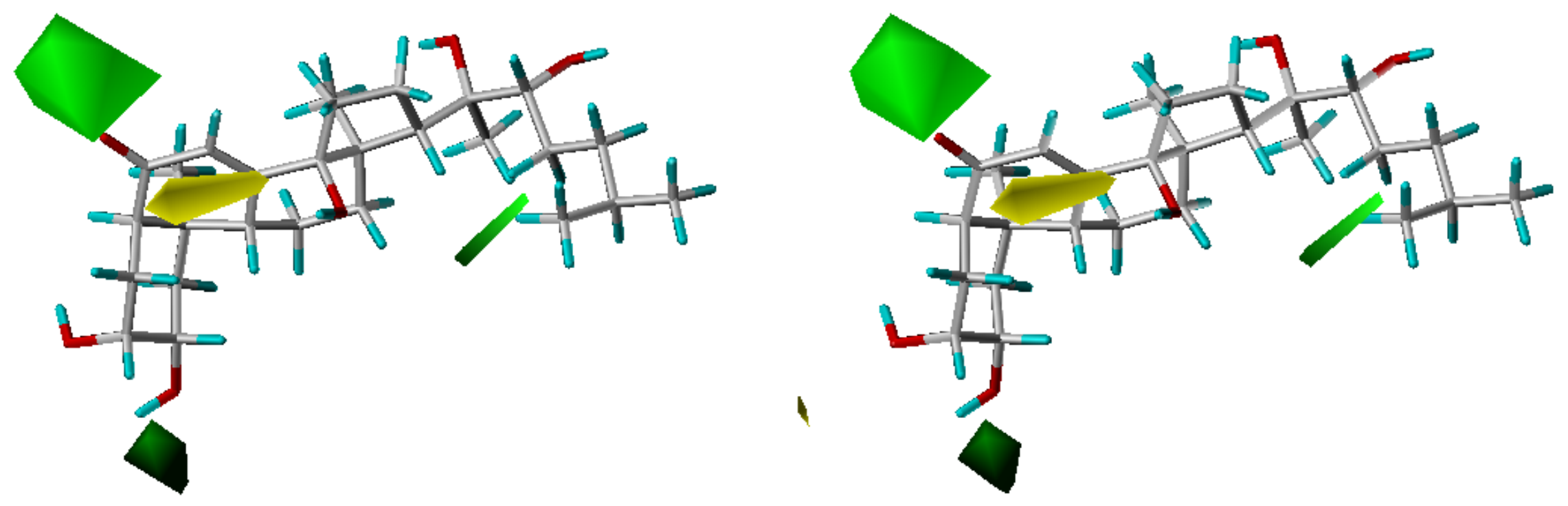


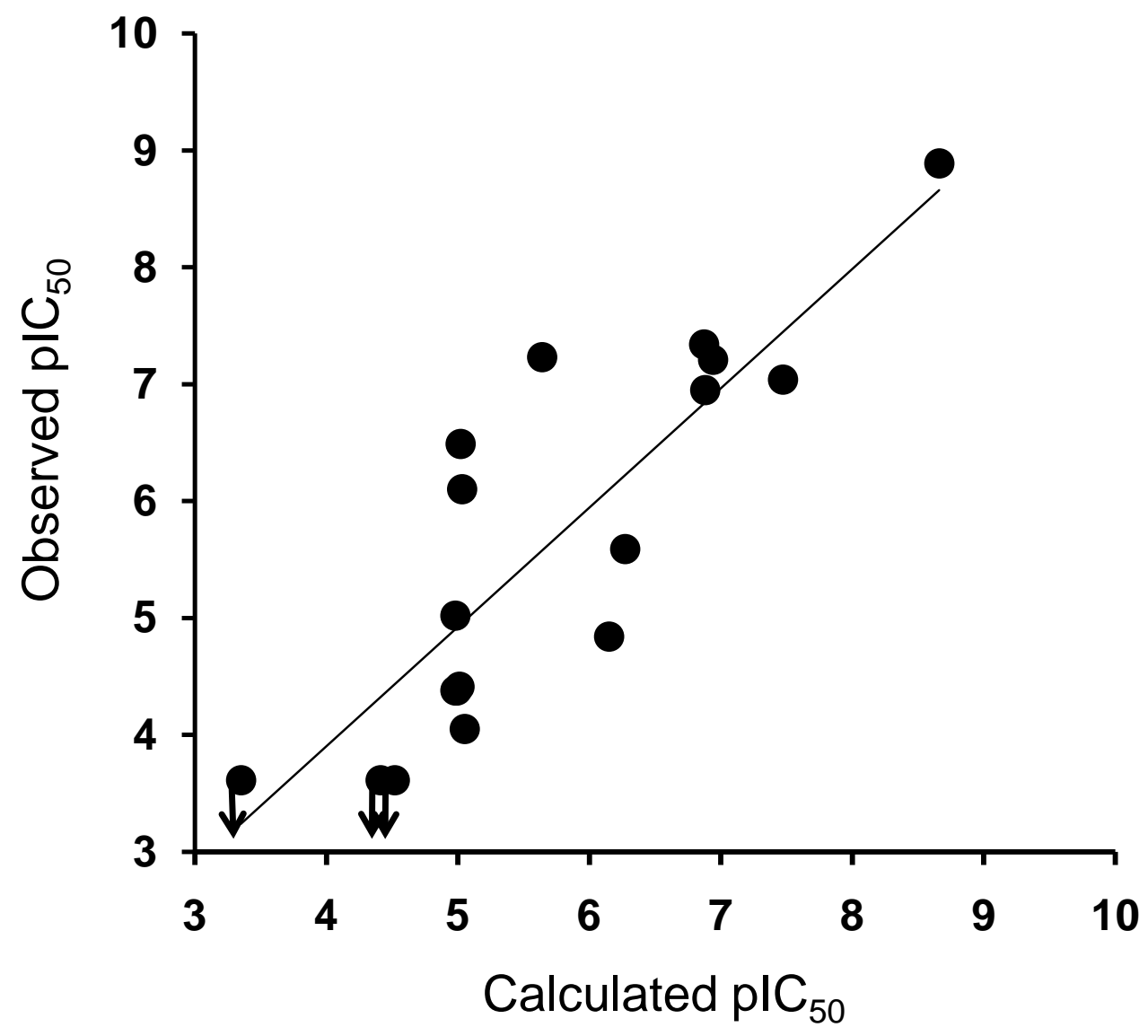


Dm 413 IPSLTYNQLA VIYKLIWYQD GYEQPSEEDL RRIMSQPDEN ESQTDVSFRH ITEITILTVQ LIVEFAKGLP 482 Hv 20 VPPLTANQKS LIARLVWYQE GYEQPSEEDL KRVTQT--W- --DSDMPFRQ ITEMTILTVQ LIVEFAKGLP 91

Dm 483 AFTKIPQEDQ ITLLKACSSE VMMLRMARRY DHSSDSIFFA NNRSYTRDSY KMAGMADNIE DLLHFCRQMF 552 Hv 92 GFAKISQSDQ ITLLKACSSE VMMLRVARRY DAATDSVLFA NNQAYTRDNY RKAGMAYVIE DLLHFCRCMY 161

Dm 553 SMKVDNVEYA LLTAIVIFSD RPGLEKAQLV EAIQSYYIDT LRIYILNRHC GDSMSLVFYA KLLSILTELR 622

Hv 162 SMMMDNVHYA LLTAIVIFSD RPGLEQPLLV EEIQRYYLNT LRVYILNQNS ASPRCAVIFG KILGILTEIR 231

Dm 623 TLGNQNAEMC FSLKLKNRKL PKFLEE IWDV H 653

Hv 232 TLGMONSNMC ISLKLKNRKL PPFLEEIWDV A 262 


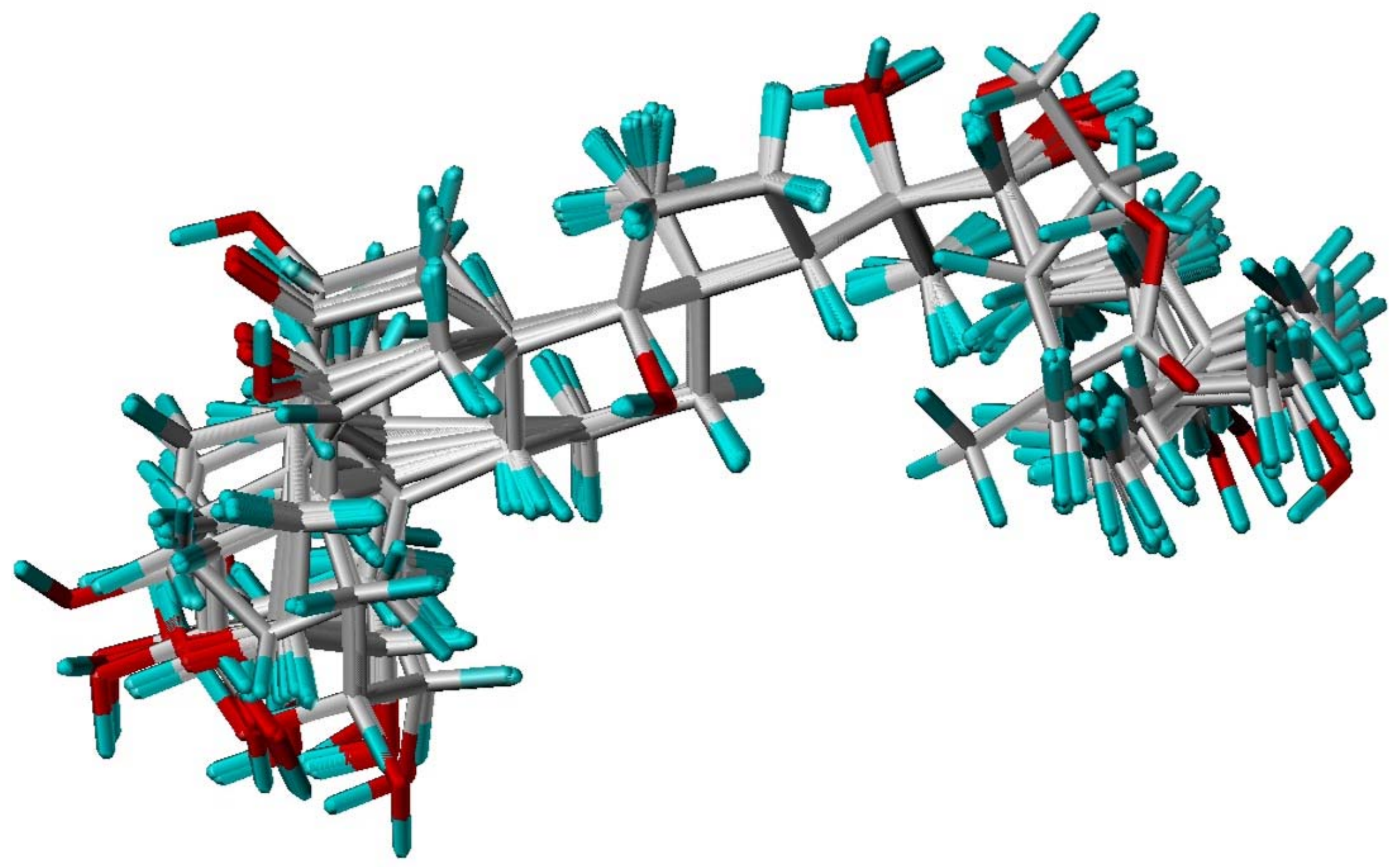

